Hydrol. Earth Syst. Sci., 17, 2107-2120, 2013

www.hydrol-earth-syst-sci.net/17/2107/2013/

doi:10.5194/hess-17-2107-2013

(c) Author(s) 2013. CC Attribution 3.0 License.

\title{
A flood episode in northern Italy: multi-model and single-model mesoscale meteorological ensembles for hydrological predictions
}

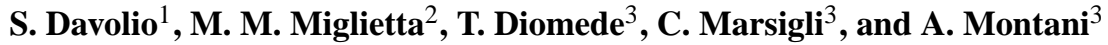 \\ ${ }^{1}$ Institute of Atmospheric Sciences and Climate, National Research Council, Bologna, Italy \\ ${ }^{2}$ Institute of Atmospheric Sciences and Climate, National Research Council, Lecce, Italy \\ ${ }^{3}$ HydroMeteoClimate Regional Service of ARPA Emilia Romagna, Bologna, Italy
}

Correspondence to: S. Davolio (s.davolio@isac.cnr.it)

Received: 5 November 2012 - Published in Hydrol. Earth Syst. Sci. Discuss.: 4 December 2012

Revised: 23 April 2013 - Accepted: 4 May 2013 - Published: 5 June 2013

\begin{abstract}
Numerical weather prediction models can be coupled with hydrological models to generate streamflow forecasts. Several ensemble approaches have been recently developed in order to take into account the different sources of errors and provide probabilistic forecasts feeding a flood forecasting system. Within this framework, the present study aims at comparing two high-resolution limited-area meteorological ensembles, covering short and medium range, obtained via different methodologies, but implemented with similar number of members, horizontal resolution (about $7 \mathrm{~km}$ ), and driving global ensemble prediction system. The former is a multi-model ensemble, based on three mesoscale models (BOLAM, COSMO, and WRF), while the latter, following a single-model approach, is the operational ensemble forecasting system developed within the COSMO consortium, COSMO-LEPS (limited-area ensemble prediction system).

The meteorological models are coupled with a distributed rainfall-runoff model (TOPKAPI) to simulate the discharge of the Reno River (northern Italy), for a recent severe weather episode affecting northern Apennines. The evaluation of the ensemble systems is performed both from a meteorological perspective over northern Italy and in terms of discharge prediction over the Reno River basin during two periods of heavy precipitation between 29 November and 2 December 2008. For each period, ensemble performance has been compared at two different forecast ranges.

It is found that, for the intercomparison undertaken in this specific study, both mesoscale model ensembles outperform the global ensemble for application at basin scale. Horizontal resolution is found to play a relevant role in modulating the
\end{abstract}

precipitation distribution. Moreover, the multi-model ensemble provides a better indication concerning the occurrence, intensity and timing of the two observed discharge peaks, with respect to COSMO-LEPS. This seems to be ascribable to the different behaviour of the involved meteorological models.

Finally, a different behaviour comes out at different forecast ranges. For short ranges, the impact of boundary conditions is weaker and the spread can be mainly attributed to the different characteristics of the models. At longer forecast ranges, the similar behaviour of the multi-model members forced by the same large-scale conditions indicates that the systems are governed mainly by the boundary conditions, although the different limited area models' characteristics may still have a non-negligible impact.

\section{Introduction}

Coupling Numerical Weather Prediction (NWP) and hydrological models is an essential practice in order to generate short- to medium-range hydrological forecasts. Moreover, it is certainly a necessary step for implementing an early warning system suitable for a medium-sized catchment (1000$10000 \mathrm{~km}^{2}$ ), characterized by complex orography and short response times. A timely prediction of the hydrological response of these river basins, suitable for emergency planning, cannot rely on observed precipitation, but needs an alternative forcing function available at earlier times (Melone et al., 2005), i.e. meteorological forecast fields. 
The provision of accurate streamflow forecasts, especially in case of flood events, represents a major research and operational challenge (Rotach et al., 2012). In such an effort, early warning systems have been developed, based on coupled state-of-the-art meteorological and hydrological models. When data from different model simulations are combined, such systems provide different scenarios and valuable probabilistic information that acknowledges the different sources of errors affecting the meteo-hydrological forecasting chains.

Although each component of the system is affected by its source of error, the available literature (Krzysztofowicz, 1999; Hapuarachchi et al., 2011; Zappa et al., 2011) seems inclined to indicate that the uncertainty affecting quantitative precipitation forecasting (QPF) is dominant. Recently, the hydrological model uncertainty was estimated ten times less pronounced than the uncertainty from rainfall forecasts (Zappa et al., 2011). Errors in QPF arise from uncertainties in the initial (and boundary) conditions and in the model formulation, growing during the forecasting process and propagating from atmospheric (rainfall) to hydrological (runoff) predictions (Zappa et al., 2010). However, several recent studies (Ranzi et al., 2009; Randrianasolo et al., 2010; Velazquez et al., 2011) showed the importance of using also hydrological ensemble prediction systems to account for the error introduced by the hydrological model, and highlighted the positive impact of data assimilation in reducing the streamflow forecast error.

In the present study the attention is focused on taking into account only the uncertainty associated with the meteorological component. Considering such problem, the main efforts for the improvement of discharge prediction have been devoted to the following: (i) development of NWP models, i.e. increasing their resolution and improving the representation of the relevant physical processes in order to attain better rainfall forecast skill (Weusthoff et al., 2010; Bauer et al., 2011), especially at the small scales that are particularly relevant for hydrological applications; (ii) development of meteorological ensemble prediction systems, which represent a suitable way to cope and deal with uncertainties, as they provide probabilistic forecasts that represent an attractive product to be used for flood predictions. Cuo et al. (2011) provide an overarching review of this topic and an up-to-date description of the main open issues related to integrated meteohydrological forecasting systems.

Ensemble prediction is a well-established practice for global meteorological models, initiated in the 1990s, since it proved to provide greater forecast skill than any single deterministic prediction (Buizza, 2008). Perturbed initial conditions, generated using either singular vectors (Palmer et al., 1997), bred vectors (Toth and Kalnay, 1997), perturbed observations in multiple data assimilation cycles (Houtekamer et al., 1996), or ensemble transform Kalman filter (Wei et al., 2006), were employed to initialize a number of different forecasts, which form all together an ensemble prediction system (EPS). More recently, multi-analysis and multi-model procedures, obtained by combining different ensemble systems, each based on a different NWP model, proved to be even more skilful (Mylne et al., 2002; Bowler et al., 2008), thus leading to the implementation of superensembles (Krishnamurti et al., 1999; Park et al., 2008) and to specific international initiatives, such as TIGGE (THORPEX Interactive Grand Global Ensemble; Bougeault et al., 2010) programme.

EPS forecasts have been used as an input for hydrological models (Gouweleeuw et al., 2005; Hamill et al., 2005; Hou et al., 2007; Thielen et al., 2009; Rotach et al., 2012), thus propagating the meteorological uncertainty along the flood forecasting system (Pappenberger et al., 2005) in order to provide a probabilistic and more informative hydrological prediction. Recently, there is a general agreement on the benefit of using ensemble forecasting (Bogner et al., 2011) even for early flood warning applications. However, although representing a progress with respect to a deterministic approach, EPSs based on global models suffer from their coarse spatial resolution and often turn out to be not accurate enough for small-basin-scale applications (Thirel et al., 2008), especially in areas characterized by complex orography. In response to such a limitation, during the last decade different ensemble approaches based on limited area models (LAMs) have been developed (Marsigli, 2009; GarciaMoya et al., 2011; Iversen et al., 2011; Montani et al., 2011) sometimes involving convection-permitting models (Davolio et al., 2008; Gebhardt et al., 2011; Vié et al., 2012). This kind of limited-area ensemble prediction systems (LEPSs), which have recently become operational in several centres, basically performs a dynamical downscaling of global EPSs and represents the state of the art for meteo-hydrological forecasting applications (Cloke and Pappenberger, 2009; Adams and Ostrowsky, 2010; Addor et al., 2011), suitable especially for risk-related events. During MAP-DPHASE (Rotach et al., 2009), the forecasters appreciated the availability of ensemble information much more than being provided with a plethora of different models. Apparently, the usual probabilistic output (probability maps, etc.), as provided by ensemble modelling systems, meets their needs (Rotach et al., 2012).

However, the accurate description of analysis and model uncertainties at the mesoscale is still an open issue, and the research is still far from assessing an optimal way for providing perturbed initial and boundary conditions to LAM ensembles (Marsigli et al., 2013). New methods of combining different LEPSs in a multi-model system are being developed; in particular, multi-analysis multi-model approaches seem able to provide a suitable way to describe the uncertainties affecting the forecasting system.

Within this framework, a meteorological ensemble system COSMO-LEPS coupled with a hydrological model (TOPKAPI) has been running operationally at ARPA-SIMC for several years, in order to provide discharge predictions for 


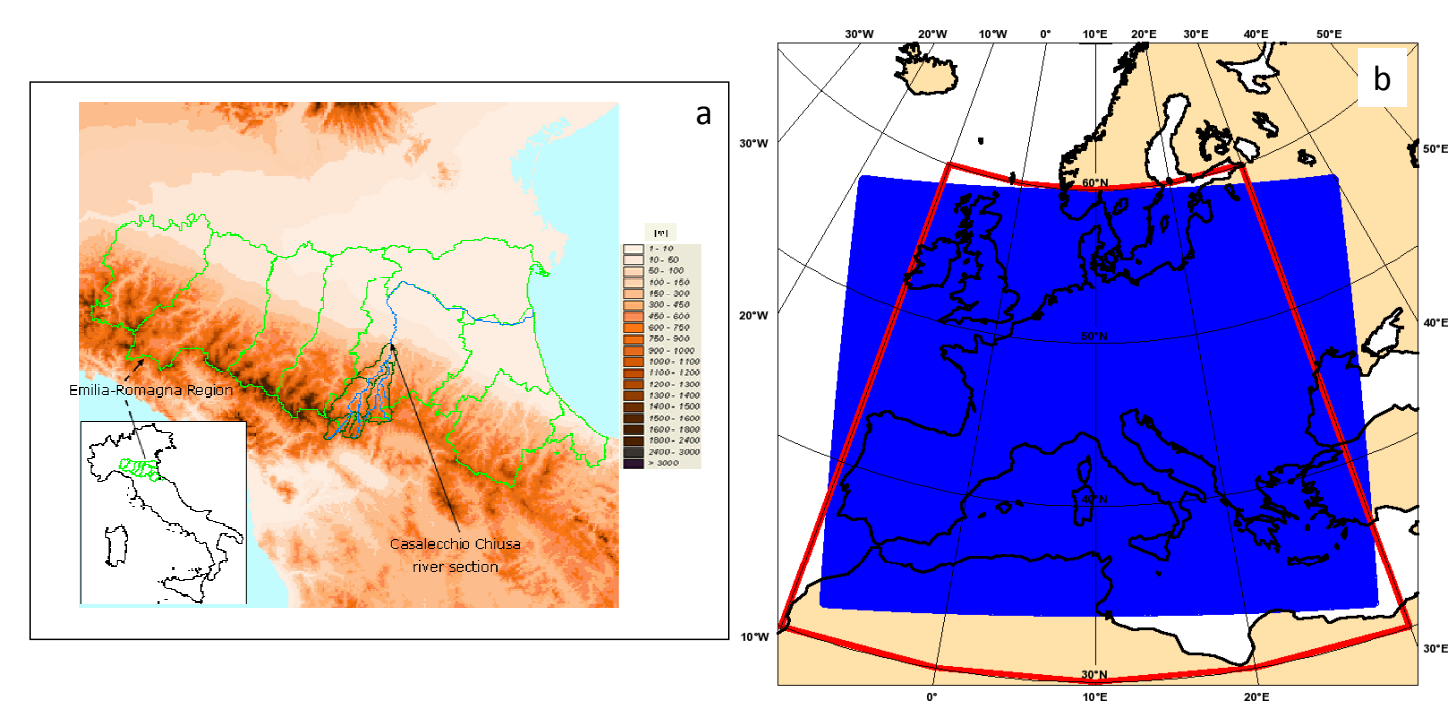

Fig. 1. (a) Localisation of the Reno River basin in the Emilia-Romagna region, northern Italy. The upper basin closure at Casalecchio Chiusa river section is indicated. (b) Model integration domains (blue area), and domain employed for the cluster analysis (red line).

civil protection purposes. Previous studies (Marsigli et al., 2008; Diomede et al., 2008b, 2009) suggested the possibility of improving the performance of this ensemble system. At the same time, collaborative research activities involving ARPA-SIMC and CNR-ISAC (Davolio et al., 2008; Diomede et al., 2008a) have been carried out, exploiting different stateof-the-art LAMs, developed or implemented in the two centres, for a multi-model approach to discharge forecasting. These activities highlighted the promising capability of the multi-model meteorological system, coupled with the hydrological model, in providing probabilistic discharge peak predictions.

Thus, it appears necessary to investigate systematically whether it is possible to improve the performance of a singlemodel ensemble (the same implemented in Addor et al., 2011), in terms of hydrological prediction, using the information that can be conveyed by an available multi-model system. Within this framework, the aim of the present paper is a comparison between the two ensemble systems for a single severe event, looking not only at the short range (as in Adams and Ostrowsky, 2010), but also at longer lead times. A case study approach clearly does not complete the investigation task, but represents just the starting point of a long and complex study.

Therefore, in the present study, two different ensemble approaches, both focused on the short-to-medium range, are compared: a multi-model ensemble, based on three LAMs developed independently, and a single-model ensemble. Both ensembles receive initial and boundary conditions from a limited number of members selected among the whole European Centre for Medium-range Weather Forecasts (ECMWF) global EPS through a clustering analysis. In order to allow for a fair comparison, the two ensembles were implemented with a similar set-up. The ensemble implementation is described in detail in Sect. 2, together with models and clustering procedure descriptions. Both the ensembles have been used to generate probabilistic precipitation maps, analysed in Sect. 3, and to provide the input fields to the same hydrological model. The results, in terms of discharge predictions, are presented in Sect. 4 and allow for the evaluation of the ensemble performance in a recent severe weather episode affecting the Reno River basin, located in northern Italy (Fig. 1) in the Apennines. The multi-model ensemble is further analysed in Sect. 5, while Sect. 6 is devoted to concluding remarks.

\section{Numerical model and ensemble generation}

The multi-model ensemble implemented here is based on three mesoscale models, BOLAM, COSMO and WRF, briefly described in the following, while the single-model approach is based on the COSMO model only (COSMO-LEPS ensemble). The two ensembles have almost the same characteristics, such as the number of members, the model horizontal resolution (about 7-8 km), and the driving global EPS (Table 1). Also, the integration domains (Fig. 1) are very similar, although the grid points are not exactly coincident. In the present section, a short description of the numerical models and of the ensembles is provided.

\subsection{BOLAM}

BOLAM (BOlogna Limited Area Model; Davolio et al., 2008) is a hydrostatic, primitive equation meteorological model with prognostic variables distributed on a nonuniformly spaced Lorenz grid. The horizontal discretization 
Table 1. Model set-up: horizontal and vertical resolutions, grid characteristic and initial/boundary conditions.

\begin{tabular}{lrccl}
\hline Model & $\begin{array}{r}\text { Horizontal } \\
\text { Resolution }\end{array}$ & $\begin{array}{c}\text { Number of } \\
\text { Grid Points }\end{array}$ & $\begin{array}{c}\text { Number of } \\
\text { Vertical Levels }\end{array}$ & $\begin{array}{l}\text { Initial/Boundary } \\
\text { Conditions }\end{array}$ \\
\hline BOLAM & $8 \mathrm{~km}$ & $426 \times 354$ & 50 & EPS (5 members) \\
COSMO & $7 \mathrm{~km}$ & $511 \times 415$ & 40 & EPS (5 members) \\
WRF & $7.5 \mathrm{~km}$ & $460 \times 380$ & 40 & EPS (5 members) \\
COSMO-LEPS & $7 \mathrm{~km}$ & $511 \times 415$ & 40 & EPS (16 members $)$ \\
\hline
\end{tabular}

uses geographical coordinates, with latitudinal rotation on the Arakawa C-grid. BOLAM uses a hybrid vertical coordinate system, in which the terrain-following sigma coordinate gradually tends to a pressure coordinate with increasing height above the ground, and with the relaxing factor prescribed as a function of the maximum orographic height present in the domain. The model implements a weighted average flux scheme for the three-dimensional advection. The temporal integration scheme is split-explicit, forwardbackward for the gravity modes. The lateral boundary conditions are imposed using a relaxation scheme that minimizes wave energy reflection. The water cycle for stratiform precipitation is described by means of five additional prognostic variables: cloud ice, cloud water, rain, snow, and graupel. Deep convection is parameterized using the Kain-Fritsch (Kain, 2004) convective scheme. The surface and boundary layer parameterization is based on the $\mathrm{E}-\mathrm{l}$ approximation, in which turbulent kinetic energy is predicted explicitly (Zampieri et al., 2005). A four-layer soil scheme is implemented for the computation of surface balances, heat and water vertical transfer, vegetation effects at the surface and in the soil, taking into account different soil types and physical parameters. The radiation is computed with a combined application of Geleyn's scheme (Ritter and Geleyn, 1992) and the ECMWF scheme.

\subsection{COSMO}

COSMO model (http://www.cosmo-model.org/; Steppeler et al., 2003) is the non-hydrostatic limited-area model of the COSMO Consortium, designed for both operational NWP and various scientific applications on the meso- $\beta$ and meso- $\gamma$ scale. COSMO is based on the primitive thermohydrodynamical equations describing compressible flow in a moist atmosphere without any scale approximation. The basic equations are written in advection form, and the continuity equation is replaced by a prognostic equation for the perturbation pressure. The model equations are solved numerically using the traditional finite difference method. A basic state, represented by a time-independent dry atmosphere at rest, is subtracted from the equations to reduce numerical errors associated with the calculation of the pressure gradient force in case of sloping coordinate surfaces. The model equations are formulated in rotated geographical coordinates and a generalized terrain following height coordinate.
The parameterization schemes used operationally are as follows: $\delta$-two-stream radiation scheme of Ritter and Geleyn (1992) for short- and long-wave fluxes, with full cloud-radiation feedback; Tiedtke (1989) mass-flux convection scheme with equilibrium closure based on moisture convergence; precipitation formation with a bulk microphysics parameterization including water vapour, cloud water, cloud ice, rain and snow with 3-D transport for the precipitating phases; prognostic turbulent kinetic energy closure at level 2.5; and multi-layer version of the Jacobsen and Heise soil model.

\subsection{WRF}

The Weather Research and Forecasting (WRF) model (see http://www.wrf-model.org; Skamarock et al., 2008) is a numerical weather prediction system that solves the fully compressible, non-hydrostatic Euler equations. The model uses the terrain-following, hydrostatic-pressure vertical coordinate with vertical grid stretching. The prognostic equations are cast in conservative (flux) form for conserved variables, while non-conserved variables like pressure and temperature are diagnosed from prognostic conserved variables. The horizontal grid is Arakawa-C.

WRF offers multiple options for physics parameterization schemes that can be selected based on the specific problem that is investigated. In the present model configuration (version ARW-3.1), the following schemes have been chosen: Thompson et al. (2004) microphysics, which includes six classes of moisture species plus number concentration for ice as prognostic variables; Kain (2004) cumulus parameterization; rapid radiative transfer model for long-wave radiation and Dudhia (1989) scheme for short-wave radiation; a turbulent kinetic energy closure, the Mellor-Yamada-Janjíc scheme, for the boundary layer; and the Noah land-surface model (Niu et al., 2011).

\subsection{Ensemble systems: COSMO-LEPS and multi-model}

COSMO-LEPS is the mesoscale limited-area ensemble developed and implemented by ARPA-SIMC in the framework of the COSMO Consortium and has been running operationally at ECMWF since November 2002 (Montani et al., 2011). The ensemble is based on 16 runs of the COSMO 
model and was designed for high-resolution probabilistic forecasts up to day five. The ensemble is generated from the global ECMWF EPS and combines the forecast potential of a high-resolution, non-hydrostatic, limited-area model with the probabilistic information of the ensemble approach. Due to the constraints on the computational resources, the methodology on which COSMO-LEPS is based reduces the number of global-ensemble elements driving the limited-area runs, but still keeps a large fraction of the driving-ensemble information. Specifically, an ensemble-size reduction is performed on 102 members of two successive ECMWF EPS runs (00:00 and 12:00 UTC of day $d$ ), since each EPS consists of one control run plus 50 perturbed members. EPS members are grouped into 16 clusters, following a cluster analysis (see Montani et al., 2011, for details) performed over the area shown in Fig. 1. From each cluster, a representative member (RM) is selected, which provides initial and boundary conditions to each COSMO model run. Moreover, for each COSMO-LEPS run the procedure chooses randomly either Kain-Fritsch or Tiedtke convection scheme, and perturbs turbulence and other physics parameterization schemes randomly.

The same clustering procedure described above is applied again for selecting $5 \mathrm{RMs}$ in order to drive the multi-model forecasting system. Since the results of the cluster analysis are different from those for COSMO-LEPS, different initial/boundary conditions force the two ensembles. For each initialization time, the multi-model is therefore based on 5 forecasts issued by each implemented LAM, producing 15 forecasts overall.

Summarizing, the main difference between the two ensembles resides in the relative importance attributed to the representation of the boundary condition error with respect to that of the LAM error. For the single-model ensemble, the same LAM was run 16 times receiving initial and boundary conditions from 16 selected members of the ECMWF EPS, while for the multi-model ensemble, only 5 EPS members were selected out of the EPS, but 3 different LAMs have been run on each EPS member. Both ensemble systems are integrated in time for $132 \mathrm{~h}$, and three initialization times $24 \mathrm{~h}$ apart have been selected: 12:00 UTC of three consecutive days, 26, 27 and 28 November 2008. Hourly rainfall fields produced by the two ensemble systems are provided to the same hydrological model TOPKAPI in order to produce ensemble discharge forecasts.

\subsection{Hydrological model: TOPKAPI}

The streamflow predictions are provided by TOPKAPI (TOPographic Kinematic APproximation and Integration) (Todini and Ciarapica, 2002), a distributed rainfall-runoff model. TOPKAPI couples the kinematic approach with the topography of the catchment and transfers the rainfall-runoff processes into three "structurally similar", zero-dimensional, non-linear reservoir equations. Three equations, which are

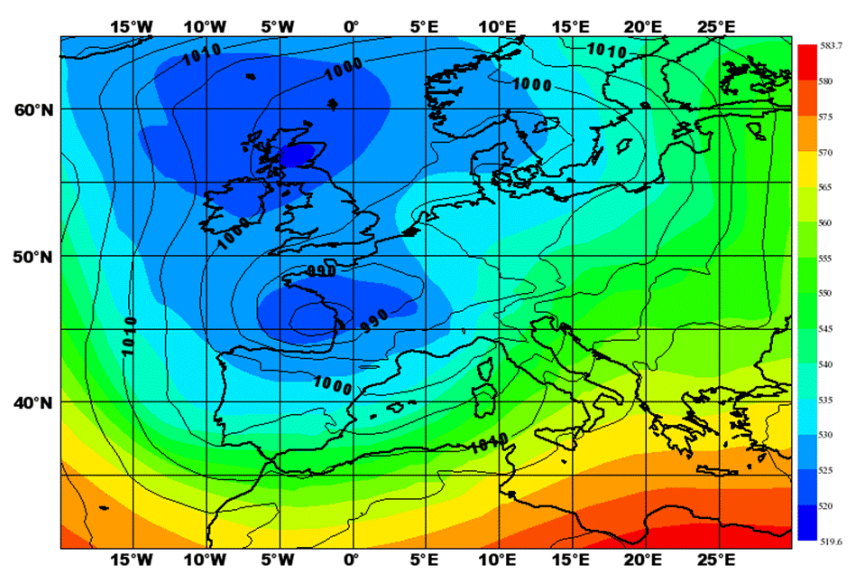

Fig. 2. ECMWF analysis at 00:00 UTC, 30 November 2008. Geopotential height at $500 \mathrm{hPa}$ (gpm, colour shading) and mean sea level pressure (hPa, contour).

derived from the integration in space of the non-linear kinematic wave model, describe the drainage in the soil, the overland flow on saturated or impervious soils, and the channel flow, respectively. The parameters of the model are shown to be scale independent and obtainable from digital elevation maps (DEMs), soil maps and vegetation or land-use maps in terms of slopes, soil permeabilities, topology and surface roughness. Land cover, soil properties and channel characteristics are assigned to each grid cell that represents a computational node for the mass and the momentum balances. The flow paths and slopes are defined starting from the DEM, according to a neighbourhood relationship based on the principle of minimum energy. The evapotranspiration is taken into account as water loss, subtracted from the soil water balance. This loss can be a known quantity, if available, or it can be calculated using temperature data and other topographic, geographic and climatic information. The snow accumulation and melting component is driven by a radiation estimate based upon air temperature measurements. A detailed description can be found in Liu and Todini (2002).

The calibration and validation procedures of TOPKAPI over the Reno River basin are based on an hourly meteohydrological data set available from 1990 to 2000. TOPKAPI is currently used for the real-time flood forecasting system operational at ARPA-SIMC.

\section{Meteorological analysis}

\subsection{Case study}

The severe weather period between 29 November and 2 December 2008 was characterized by the presence of a deep cold trough in the middle troposphere over the western Mediterranean Sea (Fig. 2). This synoptic configuration was associated with a cyclonic circulation affecting all western 


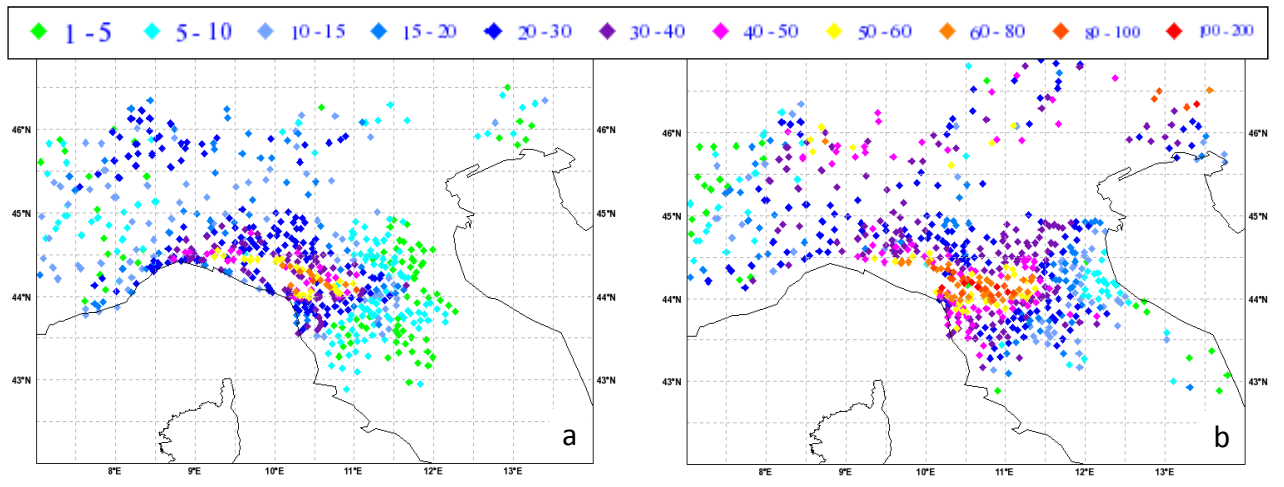

Fig. 3. Observed precipitation (mm) for the two periods of most intense rainfall: (a) $6 \mathrm{~h}$ accumulated rainfall at 00:00 UTC, 30 November 2008; (b) $24 \mathrm{~h}$ accumulated rainfall at 12:00 UTC, 1 December 2008.

and northern Europe, driving several frontal systems towards the Italian peninsula. The presence of a blocking anticyclone located over Eastern Europe, together with the meridional flow along the western side of the trough, maintained the synoptic situation nearly unchanged for several days. Intense warm and moist south-westerly flow on the eastern side of the trough, impinging on the northern Apennines, was responsible for severe weather and heavy precipitation in the area. In particular, two periods of intense precipitation (Fig. 3), during the nights of 29 November and in a $24 \mathrm{~h}$ period between 30 November and 1 December, produced two relevant discharge peaks of the Reno River, a medium-sized catchment (total dimension about $5000 \mathrm{~km}^{2}$ ), whose upstream portion (about $1000 \mathrm{~km}^{2}$ ) belongs to the north-eastern slopes of the northern Apennines. The Reno River basin has been studied in the past (Davolio et al., 2008; Diomede et al., 2008a) and was the subject of investigation in several European research projects in relation to the application of real-time flood forecasting systems. In both periods of heavy rainfall analysed in the present study, the warning threshold was exceeded at the closure section of the mountain portion of the Reno catchment, Casalecchio Chiusa, characterized by a concentration time of about $10-12 \mathrm{~h}$. In the operational practice, a flood event at such river section is defined when the water level, recorded by the gauge station, reaches or overcomes the value of $1.6 \mathrm{~m}$ (corresponding to a discharge value of about $630 \mathrm{~m}^{3} \mathrm{~s}^{-1}$ ). This value represents the warning threshold, while the alarm level is set to $2.5 \mathrm{~m}$ (corresponding to a discharge value of about $1480 \mathrm{~m}^{3} \mathrm{~s}^{-1}$ ).

\subsection{Ensemble results: probability of precipitation}

The evaluation of the ensemble systems is firstly performed from a meteorological perspective over an area larger than the single catchment (e.g. entire northern Italy). The attention is focused on the two periods of intense precipitation: $6 \mathrm{~h}$ between 29 November (18:00 UTC) and 30 November (00:00 UTC), and $24 \mathrm{~h}$ between 30 November (12:00 UTC) and 1 December (12:00 UTC). Moreover, for sake of brevity, only the simulations starting on 26 and 28 November are thoroughly analysed and discussed: thus, for each period, the ensemble performance will be compared at two different forecast ranges. For reference, global EPS results are also shown. They refer to the operational ECMWF ensemble, composed of 51 members, run at a horizontal spectral resolution $\mathrm{T}_{\mathrm{L}} 399$ (about $50 \mathrm{~km}$ ).

During the 29 November, intense precipitation in excess of $20 \mathrm{~mm} / 6 \mathrm{~h}$ (Fig. 3) affected the whole northern Apennines (with peaks close to $100 \mathrm{~mm} / 6 \mathrm{~h}$, locally) and also some Alpine areas. Results of the two LEPSs and the global EPS, in terms of probability maps of occurrence of precipitation exceeding $20 \mathrm{~mm} / 6 \mathrm{~h}$, are shown in Fig. 4, for two different forecast lead times. At longer range $(78-84 \mathrm{~h}$; initialization time 12:00 UTC, 26 November), the global EPS does not provide any indication of intense precipitation over the Reno basin, but only over western Apennines (probability up to $60 \%$ ). On the other hand, both LEPSs forecast some probability of rainfall (up to $60 \%$ for the multi-model, $30 \%$ for COSMO-LEPS) over the Reno River basin. Moreover, only the multi-model provides a signal also over the central Alps, where precipitation did occur. Similarly, for shorter forecast range (30-36 h; initialization time 12:00 UTC, 28 November), only the two LEPSs are able to forecast the possible occurrence of intense precipitation (up to $90 \%$ ) over the target basin. Very high probability is assigned to intense rainfall over western Apennines and the Alpine chain by all prediction systems, with a progressively increasing probability with shorter lead times, thus improving the confidence in the prediction as the event approaches. It is worth noting that, in the multi-model forecasts, broader areas are indicated as possibly affected by heavy precipitation, showing more uncertainty in the forecast.

Similar results have been obtained for the second period of intense precipitation. However, in this case, a longer interval of time has been considered, $24 \mathrm{~h}$ instead of $6 \mathrm{~h}$. This was chosen since the observed rainfall lasts for a longer period, and for accounting some timing errors that were evident in 

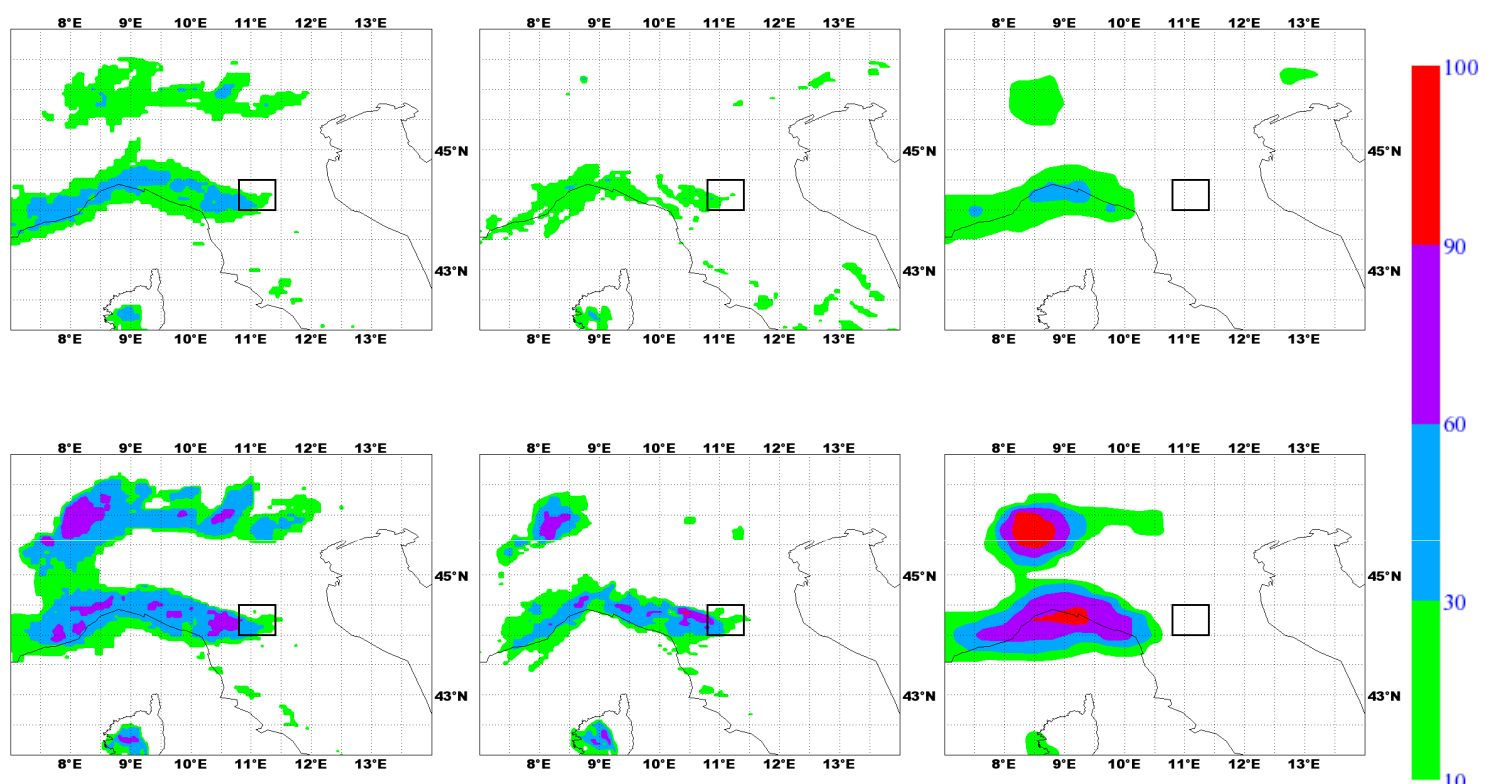

Fig. 4. Maps of probability of precipitation exceeding $20 \mathrm{~mm}$ in $6 \mathrm{~h}$ obtained at long $(+84 \mathrm{~h}$, top panels) and short ( $+36 \mathrm{~h}$, bottom panels) forecast ranges: multi-model (left), COSMO-LEPS (middle) and ECMWF global EPS (right) forecasts valid at 00:00 UTC, 30 November 2008. Reno River basin is also indicated by the black rectangle.

the precipitation forecasts, due to the much longer forecast ranges. The threshold has been increased accordingly from $20 \mathrm{~mm} / 6 \mathrm{~h}$ to $50 \mathrm{~mm} / 24 \mathrm{~h}$. Rainfall exceeding this threshold (Fig. 3) affected both the Apennines and the Alps. A nonzero probability of intense precipitation is forecast by both the ensembles, five days in advance (Fig. 5). However, only the multi-model and, partially, COSMO-LEPS are able to provide a warning for possible intense precipitation over the Reno River basin. Approaching the event, the pattern of rainfall probability does not change significantly, and still the multi-model forecasts intense rainfall over the Reno basin, with a probability ranging between 30 and $60 \%$.

While the multi-model identifies the Reno River basin as likely to be affected by intense precipitation more than three days in advance, the global EPS probability maps provide no evidence of heavy rainfall there, even at short forecast range. This result confirms that structural global model deficiencies (i.e. the low resolution and consequently the coarse representation of the orography) pose a limit to this kind of ensemble approach at such scales. Higher resolution models are needed at basin scale for medium-sized watershed, thus explaining the remarkable added value of LAM ensembles with respect to global ensembles for hydrological applications.

\section{Hydrological predictions}

The two intense precipitation events generated two relevant and distinct discharge peaks in the Reno basin (Fig. 6 top), both exceeding the warning threshold, but not reaching the alarm level. The river discharge started to increase rapidly during the night of 29 November, reaching a maximum of almost $900 \mathrm{~m}^{3} \mathrm{~s}^{-1}$ at 06:00 UTC, 30 November. A second peak, of the same magnitude, but characterized by a less steep increase of water level, occurred in the morning of 1 December. The discharge computed using rain-gauge data, spatially distributed using the Thiessen polygon method, is in good agreement with the observation at the basin closure, thus indicating that the error ascribable to the hydrological model is reasonably limited. In the following analysis, in addition to the ensemble mean, the 90th percentile is chosen as an indicator of the ensemble performance. This choice is based on previous statistical investigations (Diomede et al., 2008b, 2009) showing that, at least for COSMO-LEPS coupled with TOPKAPI, the highest quantiles $(75-90 \%)$ provide the most informative support to the forecasters in case of high-discharge events in the Reno watershed.

The ensemble discharge forecasts are strongly related to the results shown in the maps of probability of precipitation. Indeed, at longer forecast range (forecasts initialized on 26 November), discharge predictions driven by the global EPS fail to generate any relevant peak, while those driven by both LEPSs are remarkably better (Fig. 6, top panels). Although underestimated in magnitude, the possible occurrence of high discharge peaks is forecast four and five days ahead by both LEPSs. In particular, at these long forecast ranges, some members of the multi-model correctly exceed the warning threshold. Furthermore, a reasonable reproduction of the two peaks, observed $24 \mathrm{~h}$ apart, is provided by the 90th percentile of the multi-model. COSMO-LEPS displays 


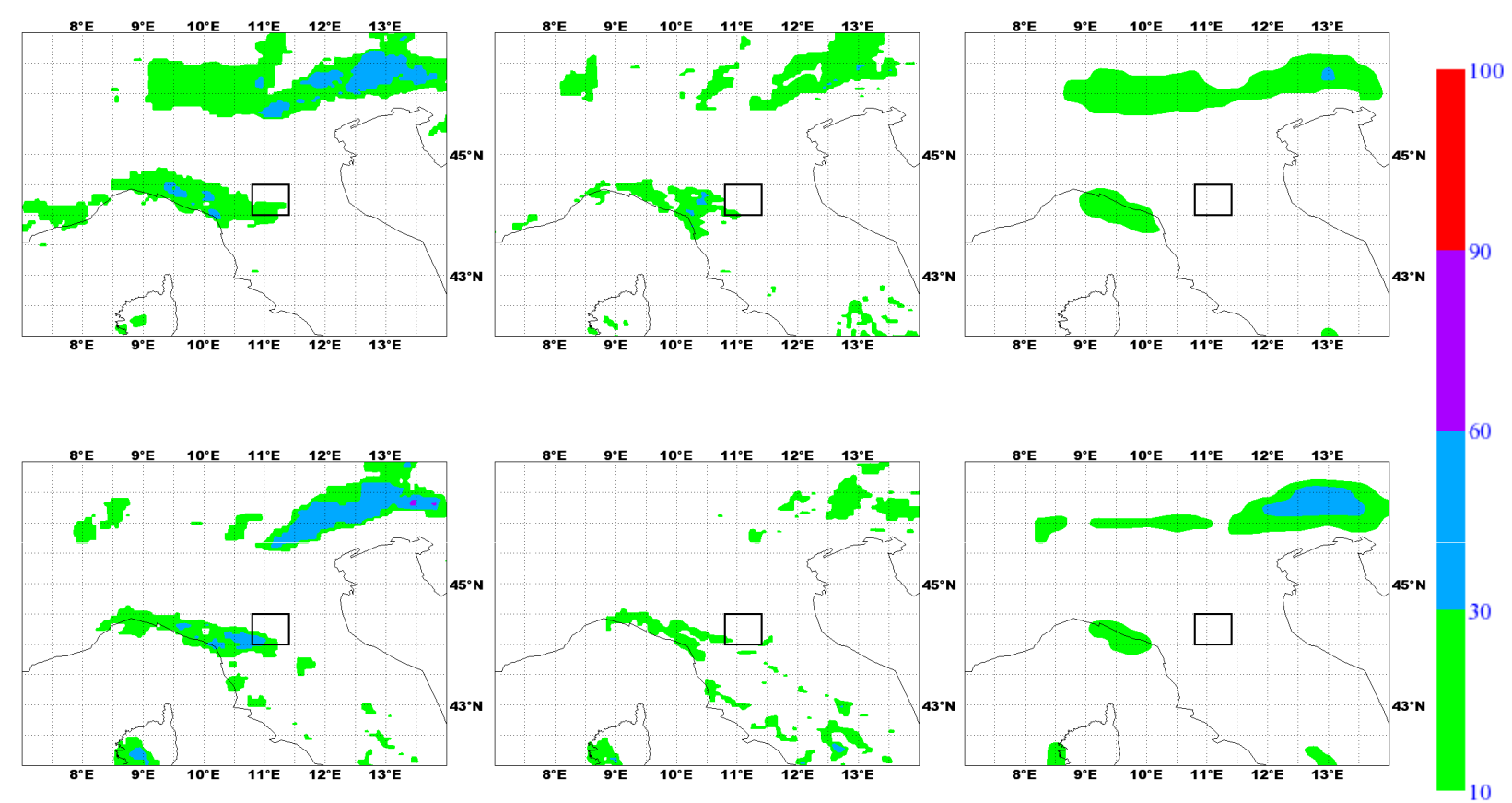

Fig. 5. Maps of probability of precipitation exceeding $50 \mathrm{~mm}$ in $24 \mathrm{~h}$ obtained at $+120 \mathrm{~h}$ (top panels) and $+72 \mathrm{~h}$ (bottom panels) forecast range: multi-model (left), COSMO-LEPS (middle) and ECMWF global EPS (right) forecasts at 12:00 UTC, 1 December 2008.
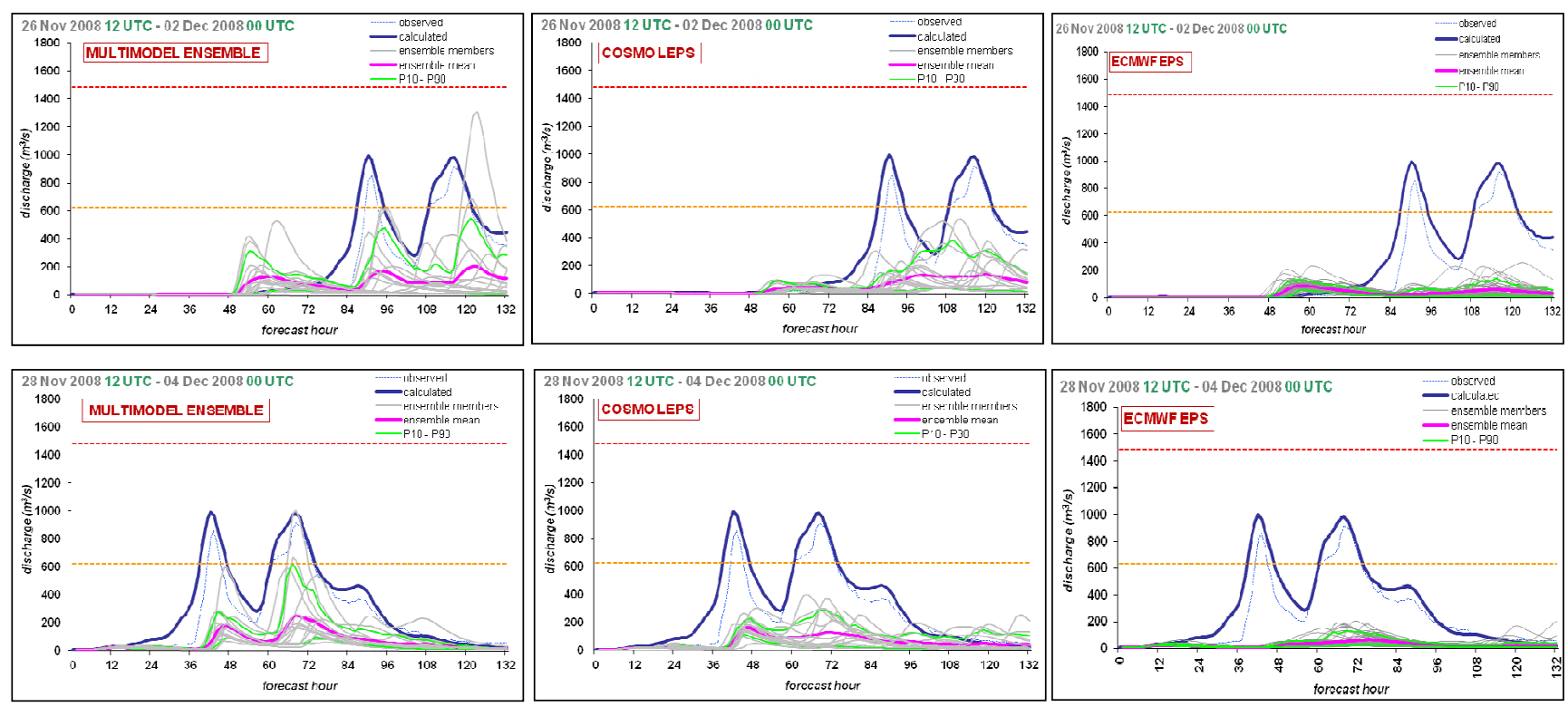

Fig. 6. Discharge forecasts $\left(\mathrm{m}^{3} \mathrm{~s}^{-1}\right.$ ) as a function of the forecast range $(h)$. The different (grey) curves have been obtained by feeding the TOPKAPI hydrological model with the precipitation forecast by the ensemble members: multi-model (left), COSMO-LEPS (middle) and ECMWF global EPS (right). The rain-gauge-driven (thick blue line) and the observed (blue dashed line) discharges are also plotted for reference. The pink line represents the ensemble mean, while the two green lines represent the 10th and the 90th percentile curves. Top panels refer to forecasts initialized at 12:00 UTC, 26 November 2008 (long range in the text); bottom panels to those initialized at 12:00 UTC, 28 November 2008 (short range in the text). Orange (red) horizontal line indicates warning (alarm) level. 

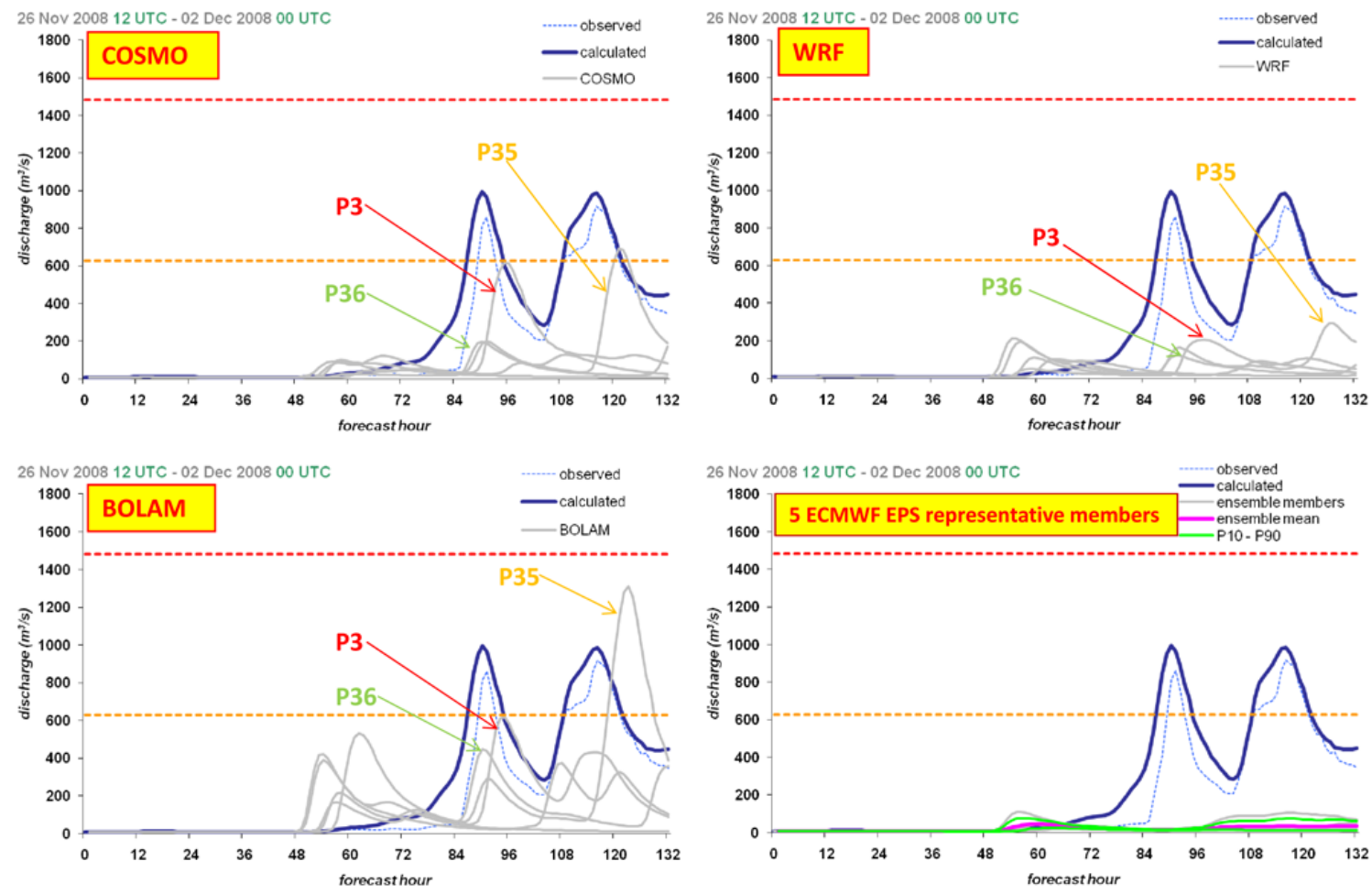

Fig. 7. Discharge forecasts $\left(\mathrm{m}^{3} \mathrm{~s}^{-1}\right)$ as a function of the forecast range $(h)$ obtained by feeding the TOPKAPI with the rainfall predicted by the five members of each model of the multi-model ensemble system and for the five representative members of the ECMWF global EPS. Forecasts are initialized at 12:00 UTC, 26 November 2008 (long range; see text). The rain-gauge-driven (thick blue line) and the observed (blue dashed line) discharges are also plotted for reference. The forecasts driven by a particular representative member of the global ensemble are indicated with arrows and with the member number. Orange (red) horizontal line indicates warning (alarm) level.

some relevant peaks, although below the warning level, and the 90th percentile does not represent the occurrence of two separate peaks.

Even at shorter forecast ranges (initialization date 28 November), up to two and three days in advance, LEPSs remarkably outperform the global EPS (Fig. 6, bottom panels). Among the ensemble systems, the discharges obtained with the multi-model display a larger spread among the members, and the 90th percentile provides a more accurate prediction, especially concerning the second peak. Also, at this range, the 90th percentile of the hydrological ensemble driven by COSMO-LEPS provides some hints of the occurrence of two peaks, although underestimating their magnitude. On the other hand, the flood event is still missed using the global EPS. Although improving the hydrological forecasts with respect to the system driven by the global ensemble, in general both LEPSs underestimate the discharge peaks, even considering the 90th percentile (Fig. 6, green line).

By analysing each curve of the multi-model ensemble forecasts at long range (Fig. 7), it is possible to recognize that the highest peaks are associated with mesoscale forecasts driven by the same global ensemble representative members (namely, members 3, 35 and 36 of the EPS). Moreover, all the meteorological forecasts driven by member 35 produce the two separate peaks in the discharge prediction, although the intensity of the peaks is significantly different among the models. It means that for longer lead times (more than 3 days), the behaviour of the different members of the multimodel is dominated by the boundary condition forcing, although the characteristics of each single LAM still have an impact at least in modulating the peak intensity. This is not true for shorter forecast ranges (not shown), where it is not possible to identify the same clear correspondence between discharge forecasts and driving representative members. In this case, the impact of the boundary conditions is weaker, and the difference among the members is reasonably ascribable to the characteristics of the single models of the ensemble.

\section{Further considerations on multi-model performance}

In order to provide some support to these conclusions and to investigate in more detail the behaviour of the multimodel ensemble, a further meteorological analysis is performed. In the following, the attention is thus focused on the multi-model results, and the precipitation fields forecast by its single members are shown for different lead times. Only 
the first period of intense precipitation (night of 29 November, Fig. 3) is considered, since it allows for the analysis of the forecast behaviour at both short $(+36 \mathrm{~h})$ and long range $(+84 \mathrm{~h})$.

At longer forecast range (simulations initialized at 12:00 UTC, 26 November), the variability of the rainfall fields (Fig. 8) among the five forecasts issued by the same LAM is larger than the variability among the forecasts issued by the three LAMs driven by the same representative member. In the latter case, although the same boundary conditions provided by the representative member tend to force the three LAMs towards a similar prediction, the different model characteristics seem able to preserve still remarkable differences in the forecasts. Therefore, in a qualitative way, it is quite simple to identify the worst forecast for each of the three LAMs as the one driven by the same global representative member (m12) (Fig. 8, second panel of each row). The three mesoscale predictions that use the initial and boundary conditions provided by this representative member are affected by a remarkable underestimation of the precipitation all over the displayed domain, both over the Apennines and over the Alps, missing completely the heavy precipitation over northern Italy and the Reno basin.

A straightforward explanation of the LAM forecast failure may be found comparing the large-scale fields of the $\mathrm{m} 12$ forecast (that drives the LAM predictions) with the ECMWF analysis, both at 18:00 UTC, 29 November 2008, corresponding to the beginning of the heavy rainfall period (Fig. 9). Indeed, the geopotential field at $500 \mathrm{hPa}$ of the $\mathrm{m} 12$ simulation presents a remarkable and evident error, displaying a westerly and slightly anti-cyclonic mid-tropospheric flow over northern Italy and in particular over the Apennines, instead of the observed south-westerly flow, typically a harbinger of heavy precipitation in the target area. Also the forecast temperature field in the lower layer does not agree with the analysis. Being driven by a forecast characterized by such a large error, at long forecast range (more than three days in advance) all the corresponding LAM forecasts are consequently affected by a similar and remarkable error too. It is worth noting that an error of the same magnitude is not present in the forecasts provided by any other representative members. Moreover, it is possible to assess that mesoscale forecasts driven by representative member m36 display a pretty good forecast.

Therefore it seems reasonable to conclude that at long forecast range (day 3-5) the forcing provided by the boundary conditions is evident in the behaviour of the multi-model ensemble. However, LAM characteristics may remarkably impact the forecast, although often to a lesser extent, and this represents the main expected added value of the multi-model approach. Indeed, BOLAM generally forecasts more intense precipitation with respect to the other two models of the ensemble. Also, small qualitative differences among the model precipitation fields are amplified in terms of hydrological response, so that pretty similar rainfall patterns, produced by the three LAMs forced by the same representative member (Fig. 8), generate significantly different discharge predictions (Fig. 7). This sensitivity of the hydrological response to small-scale rainfall pattern is a clear indication that coupled atmospheric-hydrological simulations may serve as an effective validation tool for atmospheric models at regional (or sub-regional) scale (Jasper and Kaufmann, 2003).

Repeating the analysis of the multi-model results for shorter forecast range ( $36 \mathrm{~h}$ ) during the same period of heavy rainfall (Fig. 10), the five forecasts issued by the same mesoscale model present much less variability than for long forecast range. In this case, the different forecast "trajectories", due to different initial conditions, have not fully diverged yet, since the initial perturbations have not grown enough during such a short forecast range. This is partially due to the properties of the global EPS, whose initial perturbations are optimized for the medium range, as the clustering window is between +96 and $+120 \mathrm{~h}$. Also, the large-scale fields driving the multi-model (not shown) as boundary conditions are quite close to each other and in good agreement with the global analysis, and have not fully entered the integration domain from the boundaries. At short forecast ranges, the strong similarities between the LAM forecasts driven by the same representative member (as seen for long lead times) are not present any longer, and it is not easy anymore to recognize whether a specific representative member of the global EPS drives the worst or the best forecast for all the LAMs. However, moving from one model to the other, large differences among the precipitation fields are evident. Therefore it is reasonable to speculate that the variability among the LAM forecasts is dominated by the model characteristics. This is one of the positive aspects of the multi-model, which allows for a quite large spread among the forecasts also at short ranges. Similar considerations can be drawn from the second period of intense precipitation.

\section{Conclusions and future plans}

In the present study, two different meteorological limitedarea ensemble approaches to QPF have been implemented in order to provide a range of possible meteorological scenarios to the same hydrological rainfall-runoff model: a multimodel ensemble based on three mesoscale models, BOLAM, COSMO and WRF, and a single-model approach, the COSMO-LEPS ensemble. In order to allow for a fair comparison, the two ensembles have been implemented with almost the same characteristics; also, both ensembles are driven by a limited number of members selected among those of a largescale EPS, to which the two limited-area ensembles have also been compared. The implementation of the proposed systems is presented just for a case study characterized by two periods of intense precipitation over northern Apennines, whose ground effects are evaluated over the Reno River basin, a medium-sized catchment in northern Italy. 

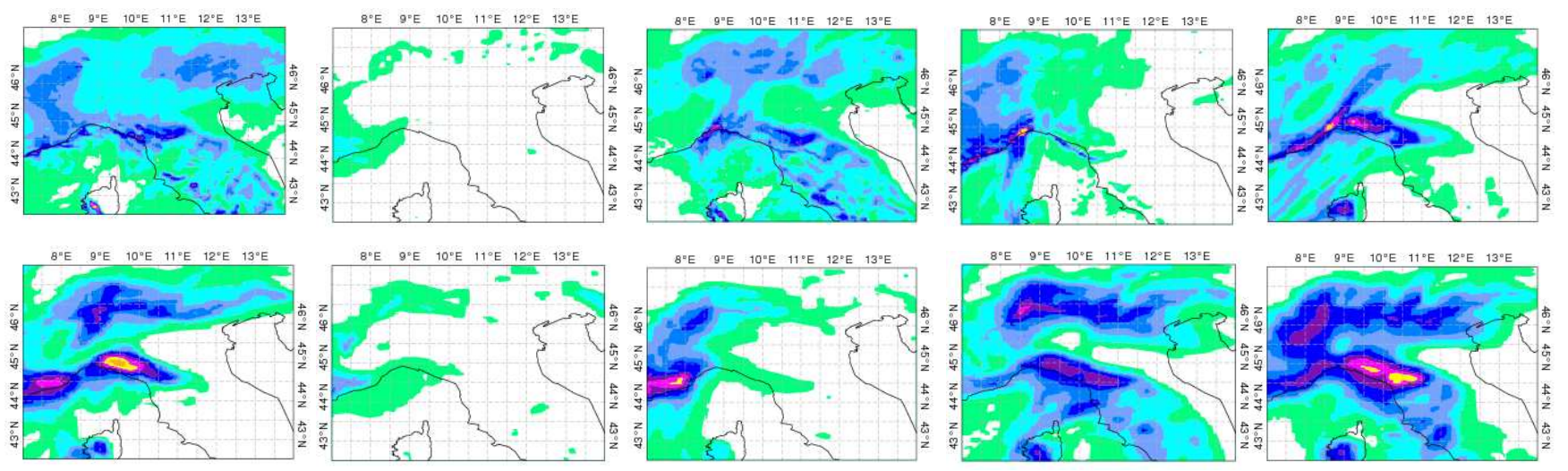

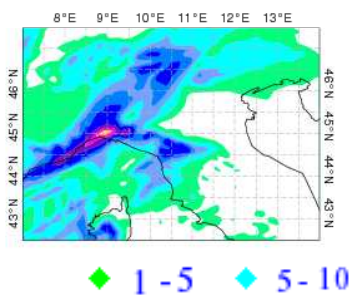

m3

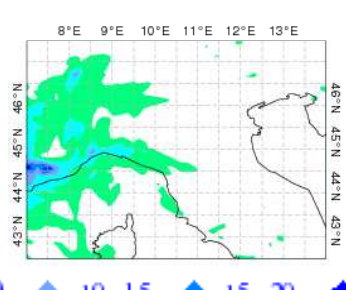

m12

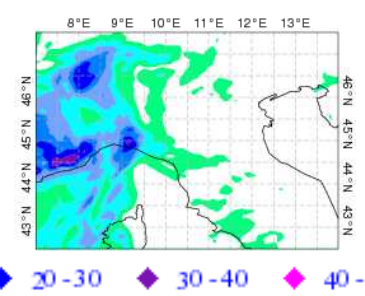

$\mathrm{m} 23$

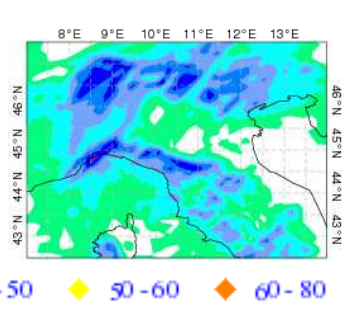

m35

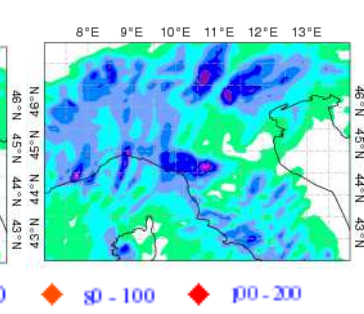

m36

Fig. 8. $6 \mathrm{~h}$ accumulated precipitation $(\mathrm{mm})$ at 00:00 UTC, 30 November 2008 forecast by the different members of the multi-model ensemble at long-range ( $+84 \mathrm{~h}$; see text). Five forecasts for each model: COSMO (top), BOLAM (middle) and WRF (bottom). Models are initialized at 12:00 UTC, 26 November 2008. The driving global representative member $(\mathrm{m})$ is indicated below each panel column.
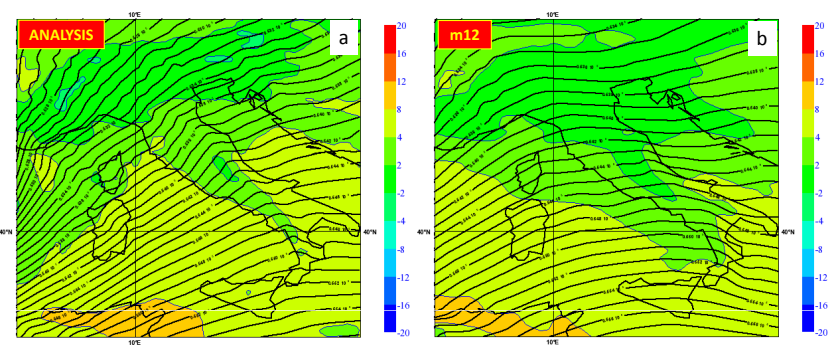

Fig. 9. Geopotential height at $500 \mathrm{hPa}$ (gpm, contour lines) and temperature at $850 \mathrm{hPa}$ (colour shading) at 18:00 UTC, 29 November 2008. (a) ECMWF analysis. (b) Forecast fields issued by the ECMWF representative member number 12 (m12).

In this study the authors mimic the use in real time of the two ensemble hydrological forecasting systems, to assess how the forecasters would use the provided information in the presence of a significant event. Within this framework, the comparison among EPSs highlights important aspects, which deserve further investigations:

1. The added value of mesoscale models with respect to the global ensemble in predicting any relevant probability of intense precipitation over the relatively small Reno River basin. Both LEPSs improve the forecast quality with respect to the "driving" global model ensemble for this case study, also in terms of discharge prediction over the Reno River basin;

2. The multi-model ensemble provides better results with respect to the single-model system. In particular, the former allows one to address the potential threat associated with the specific event discussed, correctly indicating the occurrence, intensity and timing of the two discharge peaks $24 \mathrm{~h}$ apart;

3. The progressively increasing probability at shorter lead times improves the confidence in the prediction as the event approaches. In the multi-model forecasts, the areas with high probability of heavy precipitation are generally broader, and the differences in the forecast members are larger. The 90th-percentile curve of the discharge forecasts, issued using the multi-model system coupled with TOPKAPI, is able to correctly reproduce, especially at longer range, the occurrence of two separate intense peaks. Based on the local forecasters' experience, as well as on previous statistical analysis, this would have been a piece of valuable information for the actual forecast of the flood. Using the multi-model ensemble the possible flood occurrence would have been predicted with a sufficient lead time, and the magnitude of the event could have been properly estimated by the decision makers. 

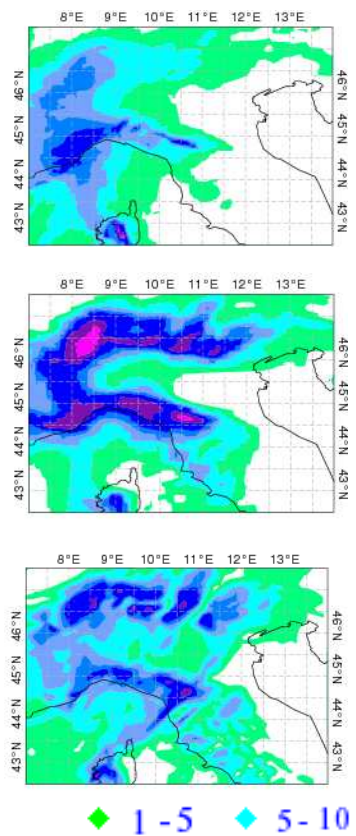

m5
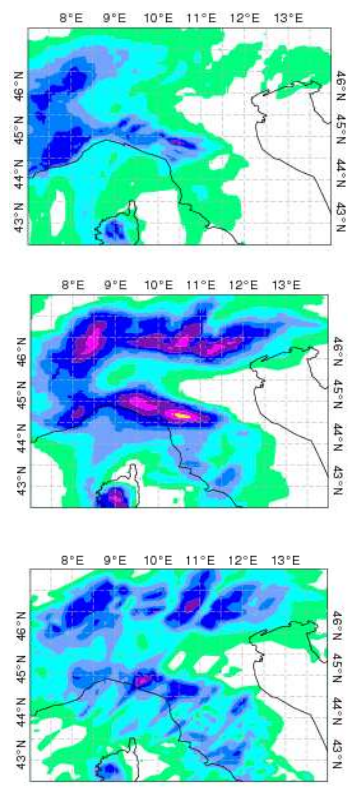

$10-15$

$\mathrm{m} 15$
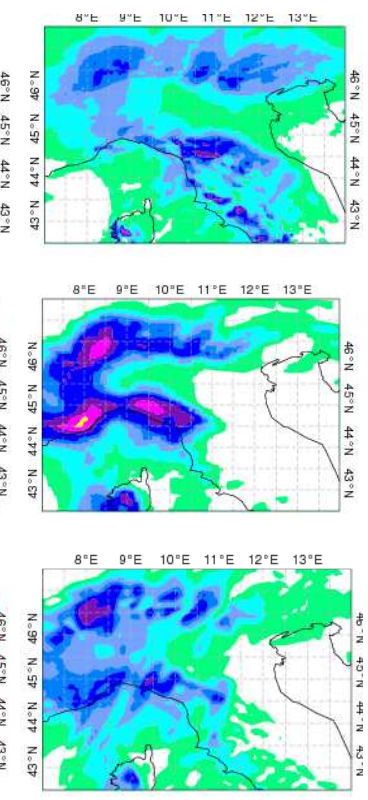

$20-30$
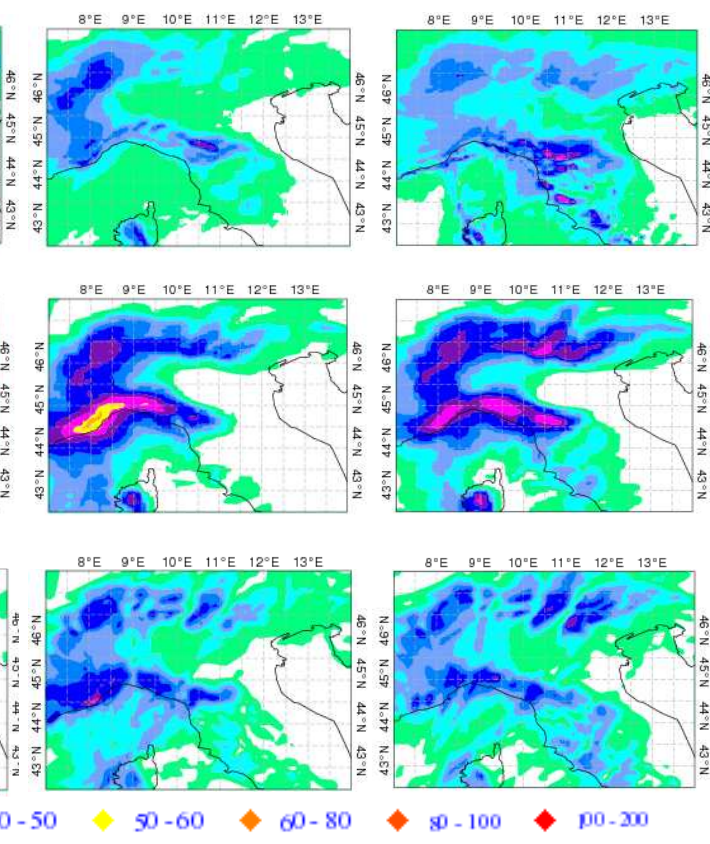

m44
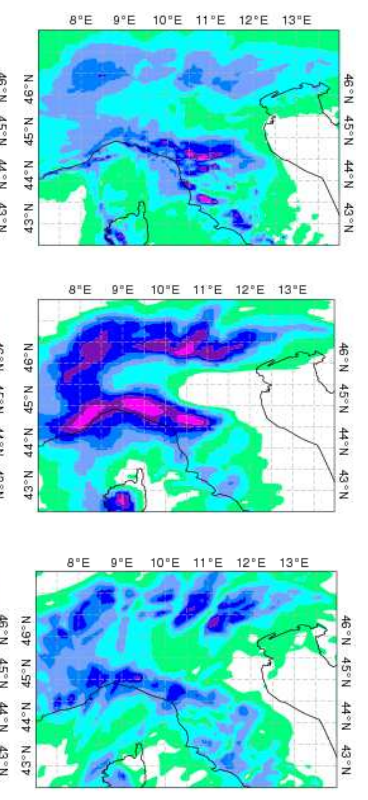

m26

Fig. 10. As in Fig. 8 but for the forecasts initialized at 12:00 UTC, 28 November 2008 (short range, +36 h; see text).

Still focusing on the multi-model ensemble, a different behaviour can be identified considering different forecast ranges, but general conclusions cannot be drawn and more cases are definitely needed to provide solid statistical basis to the results.

It is worth stressing again that the considerations of the present research are confined to just one case study, and further events, associated with different synoptic conditions, need to be analysed in order to generalize these conclusions. Also, the present paper is limited to ensembles based on convection-parameterized models. The horizontal resolution adopted here (7-8 km) is close to the "no man's land" (Weisman et al., 2008) separating classical convective parameterization schemes from explicitly convection-resolving models. As a result, the ability of mesoscale models to accurately reproduce atmospheric phenomena on such fine spatial scales can be questionable. Further simulations using short-range ensembles employing convection-resolving models at higher resolution, which should be able to better represent the small scales and to better simulate convective rainfall, will be analysed in a future study.

Acknowledgements. This work has been partly supported by the Italian Civil Protection Department in the framework of MODMET 3 project and by the Italian flagship Project RITMARE programme of CNR. The authors are also grateful to the institutions providing the rainfall observation.

Edited by: G. Grossi

\section{References}

Adams, T. and Ostrowski, J.: Short lead-time hydrologic ensemble forecasts from numerical weather prediction model ensembles. Proc. World Environment and Water Resources Congress, Providence, RI, American Society of Civil Engineers, 16-20 May 2010, 2294-2304, 2010.

Addor, N., Jaun, S., Fundel, F., and Zappa, M.: An operational hydrological ensemble prediction system for the city of Zurich (Switzerland): skill, case studies and scenarios, Hydrol. Earth Syst. Sci., 15, 2327-2347, doi:10.5194/hess-15-23272011, 2011.

Bauer, H.-S., Weusthoff, T., Dorninger, M., Wulfmeyer, V., Schwitalla, T., Gorgas, T., Arpagaus, M., and Warrach-Sagi, K.: Predictive skill of a subset of the D-PHASE multi-model ensemble in the COPS region, Q. J. Roy. Meteor. Soc., 137, 287-305, 2011.

Bogner, K., Cloke, H. L., Pappenberger, F., de Roo, A., and Thielen, J.: Improving the evaluation of hydrological multi-model forecast performance in the upper Danube catchment, Int. J. River Basin Manag., 10, 1-12, 2011.

Bougeault, P., Toth, Z., Bishop, C., Brown, B., Burridge, D., Chen, D. H., Ebert, B., Fuentes, M., Hamill, T. M., Mylne, K., Nicolau, J., Paccagnella, T., Park, Y. Y., Parsons, D., Raoult, B., Schuster, D., Dias, P. S., Swinbank, R., Takeuchi, Y., Tennant, W., Wilson, L., and Worley, S.: The THORPEX Interactive Grand Global Ensemble, B. Am. Meteorol. Soc., 91, 1059-1072, 2010.

Bowler, N. E., Arribas, A., and Mylne, K. R.: The benefits of multianalysis and poor man's ensembles, Mon. Weather Rev., 136, 4113-4129, 2008.

Buizza, R.: The value of probabilistic prediction, Atmos. Sci. Lett., 9, 36-42, 2008. 
Cloke, H. L. and Pappenberger, F.: Ensemble flood forecasting: a review, J. Hydrol., 375, 613-626, 2009.

Cuo, L., Pagano, T. C., and Wang, Q. J.: A review of quantitative precipitation forecasts and their use in short- to medium-range streamflow forecasting, J. Hydrometeorol., 12, 713-728, 2011.

Davolio, S., Miglietta, M. M., Diomede, T., Marsigli, C., Morgillo, A., and Moscatello, A.: A meteo-hydrological prediction system based on a multi-model approach for precipitation forecasting, Nat. Hazards Earth Syst. Sci., 8, 143-159, doi:10.5194/nhess-8143-2008, 2008.

Diomede, T., Davolio, S., Marsigli, C., Miglietta, M. M., Moscatello, A., Papetti, P., Paccagnella, T., Buzzi, A., and Malguzzi, P.: Discharge prediction based on multi-model precipitation forecasts, Meteorol. Atmos. Phys., 101, 245-265, 2008a.

Diomede, T., Marsigli, C., Montani, A., and Paccagnella, T.: A limited-area ensemble prediction system to drive a flood forecasting chain, Proc. of HydroPredict'2008, International Interdisciplinary Conference on Predictions for Hydrology, Ecology, and Water Resources Management - Using Data and Models to Benefit Society, Prague, edited by: Bruthans, J., Kovar, K., and Hrkal, Z., Czech Republic, 15-18 September 2008, 211214, ISBN 978-80-903635-3-3 publication of the Czech Association of Hydrogeologists, available at: http://web.natur.cuni.cz/ hydropredict 2008, 2008b.

Diomede T., Marsigli, C., Montani, A., and Paccagnella, T.: Streamflow ensemble forecast driven by COSMO-LEPS for small-size catchments in northern Italy. Proc. HEPEX Workshop on Post-Processing and Downscaling Atmospheric Forecasts for Hydrologic Applications, Toulouse, France, 15-18 June 2009, p. 6, available at: http://hepex.nmpi.net/files/download/ workshops/post-processing/Book_Abstracts.pdf), 2009.

Dudhia, J.: Numerical study of convection observed during the winter monsoon experiment using a mesoscale two-dimensional model, J. Atmos. Sci., 46, 3077-3107, 1989.

Garcia-Moya, J. A., Callado, A., Escribà, P., Santos, C., SantosMuñoz, D., and Simarro, J.: Predictability of short-range forecasting: a multimodel approach, Tellus A, 63, 550-563, 2011.

Gebhardt, C., Theis, S. E., Paulat, M., and Ben Bouallègue, Z.: Uncertainties in COSMO-DE precipitation forecasts introduced by model perturbations and variation of lateral boundaries, Atmos. Res., 100, 168-177, 2011.

Gouweleeuw, B. T., Thielen, J., Franchello, G., De Roo, A. P. J., and Buizza, R.: Flood forecasting using medium-range probabilistic weather prediction, Hydrol. Earth Syst. Sci., 9, 365-380, doi:10.5194/hess-9-365-2005, 2005.

Hamill, T., Clark, M., Schaake, J., and Buizza, R.: Second HEPEX Workshop Summary Report, Boulder, Colorado, available at: http://hepex.nmpi.net/files/download/workshops/ 2nd/HEPEX05-Summary.pdf (last access: 30 May 2013), 2005.

Hapuarachchi, H. A. P., Wang, Q. J., and Pagano, T. C.: A review of advances in flash flood forecasting, Hydrol. Process., 25, 27712784, 2011.

Hou, D., Mitchell, K., Toth, Z., Lohmann, D., and Wei, H.: Ensemble streamflow forecasting with the coupled GFS-NOAH modelling system, 3rd HEPEX workshop, Book of Abstracts, edited by: Thielen, J., Bartholmes, J., and Schaake, J., European Commission EUR22861EN, 65-68, 2007.

Houtekamer, P. L., Derome, J., Ritchie, H., and Mitchell, H. L: A system simulation approach to ensemble prediction, Mon.
Weather Rev., 124, 1225-1242, 1996.

Iversen, T., Deckmyn, A., Santos, A., Sattler, K., Bremnes, J. B., Feddersen, H., and Frogner, L. L.: Evaluation of GLAMEPS - A proposed multimodel EPS for short range forecasting, Tellus A, 63, 513-530, 2011.

Jasper, K. and Kaufmann, P.: Coupled runoff simulations as validation tools for atmospheric models at the regional scale, Q. J. Roy. Meteor. Soc., 129, 673-692, 2003.

Kain, J. S.: The Kain-Fritsch convective parameterization: An update, J. Appl. Meteorol., 43, 170-181, 2004.

Krishnamurti, T. N., Kistawal, C. M., LaRow, T. E., Bachiochi, D. R., Zhang, Z., Williford, C. E., Gadgil, S., and Surendran, S: Improved weather and seasonal climate forecasts from multimodel superensemble, Science, 285, 1548-1550, 1999.

Krzysztofowicz, R.: Bayesian theory of probabilistic forecasting via deterministic hydrologic model, Water Resour. Res., 35, 2739 2750, 1999.

Liu, Z. and Todini, E.: Towards a comprehensive physically-based rainfall-runoff model, Hydrol. Earth Syst. Sci., 6, 859-881, doi:10.5194/hess-6-859-2002, 2002.

Marsigli, C.: COSMO-SREPS Priority Project "Short Range Ensemble Prediction System (SREPS): final report, COSMO Technical report, 13, available at: http://www.cosmo-model.org/ content/model/documentation/techReports/default.htm (last access: 30 May 2013), 2009.

Marsigli, C., Montani, A., and Paccagnella, T.: A spatial verification method applied to the evaluation of high-resolution ensemble forecasts, Meteorol. Appl., 15, 125-143, 2008.

Marsigli, C., Montani, A., and Paccagnella, T.: Perturbation of initial and boundary conditions for limited-area ensemble: multimodel versus single-model approach, Q. J. Roy. Meteor. Soc., doi:10.1002/qj.2128, online first, 2013.

Melone, F., Barbetta, S., Diomede, T., Perucacci, S., Rossi, M., Tessarollo, A., and Verdecchia, M.: Review and selection of hydrological models - Integration of hydrological models and meteorological inputs, Resulting from WP1, Action 13, RISK AWARE - INTERREG III B - CADSES, 34 pp., 2005.

Montani, A., Cesari, D., Marsigli, C., and Paccagnella, T.: Seven years of activity in the field of mesoscale ensemble forecasting by the COSMO-LEPS system: main achievements and open challenges, Tellus A, 63, 605-624, 2011.

Mylne, K. R., Evans, R. E., and Clark, R. T.: Multi-model multianalysis ensemble in quesi-operational medium-range forecasting, Q. J. Roy. Meteor. Soc., 128, 361-384, 2002.

Niu, G.-Y., Yang, Z.-L., Mitchell, K. E. , Chen, F., Ek, M. B., Barlage, M., L. Longuevergne, L., Kumar, A., Manning, K., Niyogi, D., Rosero, E., Tewari, M., and Xia, Y.: The community Noah land surface model with multiparameterization options (Noah-MP): 1. Model description and evaluation with local-scale measurements, J. Geophys. Res., 116, D12109, doi:10.1029/2010JD015139, 2011.

Palmer, T. N., Barkmeijer, J., Buizza, R., and Petroliagis, T.: The ECMWF ensemble prediction system, Meteorol. Appl., 4, 301304, 1997.

Pappenberger, F., Beven, K. J., Hunter, N. M., Bates, P. D., Gouweleeuw, B. T., Thielen, J., and de Roo, A. P. J.: Cascading model uncertainty from medium range weather forecasts $(10$ days) through a rainfall-runoff model to flood inundation predictions within the European Flood Forecasting System (EFFS), Hy- 
drol. Earth Syst. Sci., 9, 381-393, doi:10.5194/hess-9-381-2005, 2005.

Park, Y. Y., Buizza, R., and Leutbecher, M.: TIGGE: preliminary results on comparing and combining ensembles, Q. J. Roy. Meteor. Soc., 134, 2029-2050, 2008.

Randrianosolo, A., Ramos, M. H., Thirel, G., Andréassian, V., and Martin, E.: Comparing the scores of hydrological ensemble forecasts issued by two different hydrological models, Atmos. Sci. Lett., 11, 100-107, 2010.

Ranzi, R., Bacchi, B., Ceppi, A., Cislaghi, M., Ehret, U., Jaun, S., Marx, A., Hegg, C., and Zappa, M.: Real-time demonstration of hydrological ensemble forecasts in MAP D-PHASE, La-HuilleBlanche, 5, 95-103, doi:10.1051/lhb/2009061, 2009.

Ritter, B. and Geleyn, J. F.: A comprehensive radiation scheme for numerical weather prediction models with potential applications in climate simulations, Mon. Weather Rev., 120, 303-325, 1992.

Rotach, M. W., Ambrosetti, P., Ament, F., Appenzeller, C., Arpagaus, M., Bauer, M. S., Behrendt, A., Bouttier, F., Buzzi, A., Corazza, M., Davolio, S., Denhard, M., Dorninger, M., Fontannaz, L., Frick, J., Fundel, F., Germann, U., Gorgas, T., Hegg, C., Hering, A., Keil, C., Liniger, M. A., Marsigli, C., McTaggartCowan, R., Montani, A., Mylne, K., Ranzi, R., Richard, E., Rossa, A., Santos-Muñoz, D., Schär, C., Seity, Y., Staudinger, M., Stoll, M., Volkert, H., Walser, A., Wang, Y., Werhahn, J., Wulfmeyer, V., and Zappa, M.: MAP D-PHASE: Real-time Demonstration of Weather Forecast Quality in the Alpine Region, B. Am. Meteorol. Soc., 90, 1321-1336, 2009.

Rotach, M. W., Arpagaus, M., Dorninger, M., Hegg, C., Montani, A., and Ranzi, R.: Uncertainty propagation for flood forecasting in the Alps: different views and impacts from MAP D-PHASE, Nat. Hazards Earth Syst. Sci., 12, 2439-2448, doi:10.5194/nhess-12-2439-2012, 2012.

Skamarock, W. C., Klemp, J. B., Dudhia, J., Gill, D. O., Barker, D. M., Duda, M. G., Huang, X.-Y., Wang, W., and Powers, J. G.: A Description of the Advanced Research WRF Version 3. NCAR Technical note NCAR/TN-475+STR, 2008.

Steppeler, J., Doms, G., Schättler, U., Bitzer, H. W., Gassmann, A., Damrath, U., and Gregoric, G.: Meso-gamma scale forecasts using the nonhydrostatic model LM, Meteorol. Atmos. Phys., 82, 75-96, 2003.

Thielen, J., Bartholmes, J., Ramos, M.-H., and de Roo, A.: The European Flood Alert System - Part 1: Concept and development, Hydrol. Earth Syst. Sci., 13, 125-140, doi:10.5194/hess-13-1252009, 2009.

Thirel, G., Rousset-Regimbeau, F., Martin, E., and Habets, F.: On the impact of short-range meteorological forecasts for ensemble streamflow predictions, J. Hydrometeorol., 9, 1301-1317, 2008.
Thompson, G., Rasmussen, R. M., and Manning, K.: Explicit forecasts of winter precipitation using an improved bulk microphysics scheme. Part I: Description and sensitivity analysis, Mon. Weather Rev., 132, 519-542, 2004.

Tiedtke, M.: A comprehensive mass flux scheme for cumulus parameterization in large-scale models, Mon. Weather Rev., 117, 1779-1800, 1989.

Todini, E. , and Ciarapica, L.: The TOPKAPI model, in: Mathematical models of large watershed hydrology, edited by: Singh, V. P. and Frevert, D. K., Littleton, Colorado, USA, Water Resources Publications, 914 pp., 2002.

Toth, Z., and Kalnay, E.: Ensemble forecasting at NCEP and the breeding method, Mon. Weather Rev., 125, 3297-3319, 1997.

Velázquez, J. A., Anctil, F., Ramos, M. H., and Perrin, C.: Can a multi-model approach improve hydrological ensemble forecasting? A study on 29 French catchments using 16 hydrological model structures, Adv. Geosci., 29, 33-42, doi:10.5194/adgeo29-33-2011, 2011.

Vié, B., Molinié, G., Nuissier, O., Vincendon, B., Ducrocq, V., Bouttier, F., and Richard, E.: Hydro-meteorological evaluation of a convection-permitting ensemble prediction system for Mediterranean heavy precipitating events, Nat. Hazards Earth Syst. Sci., 12, 2631-2645, doi:10.5194/nhess-12-2631-2012, 2012.

Wei, M. Z., Toth, Z., Wobus, R., Zhu, Y. J., Bishop, C. H., and Wang, X. G.: Ensemble transform Kalman filter-based ensemble perturbations in an operational global prediction system at NCEP, Tellus A, 58, 28-44, 2006.

Weisman, M. L., Davies, C., Wang, W., Manning, K. W., and Klemp, J. B.: Experiences with 0-36-h explicit convective forecasts with the WRF-ARW model, Weather Forecast., 23, 407437, 2008.

Weusthoff, T., Ament, F., Arpagaus, M., and Rotach, M. W.: Assessing the benefits of convection permitting models by Neighborhood Verification - examples from MAP D-PHASE, Mon. Weather Rev., 138, 3418-3433, 2010.

Zampieri, M., Malguzzi, P., and Buzzi, A.: Sensitivity of quantitative precipitation forecasts to boundary layer parameterization: a flash flood case study in the Western Mediterranean, Nat. Hazards Earth Syst. Sci., 5, 603-612, doi:10.5194/nhess-5-6032005, 2005.

Zappa, M., Beven, K. J., Bruen, M., Cofino, A. S., Kok, K., Martin, E., Nurmi, P., Orfila, B., Roulin, E., Schröter, K., Seed, A., Szturc, J., Vehviläinen, B., Germann, U., and Rossa, A.: Propagation of uncertainty from observing systems and NWP into hydrological models: COST-731 Working Group 2, Atmos. Sci. Lett., 11, 83-91, 2010.

Zappa, M., Jaun, S., Germann, U., Walser, A., and Fundel, F.: Superposition of three sources of uncertainties in operational flood forecasting chains, Atmos. Res., 100, 246-262, 2011. 